\title{
The shifting model in clinical diagnostics: how next-generation sequencing and families are altering the way rare diseases are discovered, studied, and treated
}

\author{
Matthew Might, $\mathrm{PhD}^{1}$ and Matt Wilsey, $\mathrm{MBA}^{2}$
}

We are the fathers of two patients with a newly diagnosed syndrome that is highlighted in the study by Enns et al. ${ }^{1}$ Our children are two among a handful of others in the world with this disease caused by mutations in the NGLY1 gene. It is the first recognized disorder of deglycosylation. We fully anticipate that NGLY1 will generate many interesting studies for years to come, but promoting NGLY1 is not our aim here. Instead, we would like to provide you with our perspective on a shift that is occurring in clinical diagnostics. Families of children with serious genetic diseases often enter a diagnostic odyssey, moving from gene to gene in the hope of finding an explanation for the condition. Two new developments in genetics promise to dramatically shorten the time to reach a successful diagnosis: next-generation sequencing (NGS) and family engagement through social media. The very speed with which Need et al. ${ }^{2}$ and Enns et al. ${ }^{1}$ were published suggests a new model for clinicians and researchers. In this model, families, patients, and scientists work jointly to find new patients, confirm or refute hypotheses, exchange clinical information, enhance collaboration methods, and support research toward understanding and treatment.

Our diagnostic quest started in the Fall of 2010, when a team at Duke University sequenced one of our children in an effort to measure the efficacy of exome sequencing in patients whose diagnostic journeys had become intractable. The team concluded that NGLY1 could be causal. This was game changing because the gene had not been implicated in any prior condition. It was difficult to know for sure whether the mutations Duke found were causal or not because there was only one affected patient. Beyond this limitation, many geneticists have raised concern that weak standards in declaring genetic diagnoses using NGS data could do real clinical harm. So why did the Duke team communicate with the family in the absence of a definitive diagnosis? They felt the family could understand this uncertainty (as many families can) and that sharing the NGLY1 suspicion would make the family partners in the discovery efforts. Following a demonstration in a molecular biology laboratory that the mutations found in the child eliminated the protein associated with the NGLY1 gene (this does not happen in the general population), Need et al. ${ }^{2}$ shared their nomination of NGLY1 as a likely (but by no means certain) cause of the condition.

Until very recently, the fragmented distribution of patients across institutions hindered the discovery of new rare diseases. Clinicians working with a single, isolated patient could steadily eliminate known disorders but do little more. Families would seek clinicians with the longest history and largest clinic volume to increase their chances of finding a second case, but what does a physician do when $N=1$ or if the phenotype is inconsistent across patients? These challenges are driving an increase in the use of NGS. Yet this technological advance presents new challenges of its own. Perhaps the most daunting, in our opinion, is the inability to share sequencing data quickly and universally. Standards and bioinformatic tools are needed that allow for a national repository where families or scientists can bring clinical results and NGS data for comparison. This challenge can be circumvented by tools already created for and by the Internet and social media.

To illustrate our point, six of the eight patients presented in the accompanying article were linked together after parents, physicians, or scientists working on isolated cases searched online for "NGLY1." They found a blog post ${ }^{3}$ describing the disorder written by the parents of the first confirmed patient. The blog chronicles the boy's journey (initial evaluation, visits to multiple specialists, incorrect diagnoses, and ultimately the discovery of heterozygous mutations in NGLY1). It was this personal account that allowed the ordering physician, who had been tracking a second patient with NGLY1 variants, to feel confident that the two patients were suffering from the same disorder. Another patient was discovered, on a distant continent, when a parent's Internet search for his/her child's symptoms stumbled upon the aforementioned blog. This prompted the parents to suggest targeted NGLY1 sequencing to their child's physician. Parent/patient-to-physician collaboration such as this is remarkable and is likely happening in other rare diseases with the advent of NGS.

As untrained people, we are not qualified to analyze wholeexome/whole-genome data. We cannot develop a therapeutic 
compound. We cannot design a diagnostic assay. That being said, parents can offer observations and ideas, and we can push for solutions. Nineteen months after the initial report by Need et al., ${ }^{2}$ five viable approaches to treatment are under active consideration, thanks to relentless digging by afflicted families. One parent found a compound that seems to have measurably raised the quality of life in one NGLY1 child. Another parent read about a novel (but relevant) fluorescent assay and shared it with the NGLY1 team. The team had not heard about it, but it has become a fundamental tool in the functional analysis of NGLY1. One parent has formed and funded a multi-institutional network of researchers to tackle specific projects. The capabilities of parents and the social media are frequently underestimated; we are here to say: join us! As the discovery of new diseases explodes with the deployment of NGS, we hope clinicians will consider this model seriously.

It would be easy to write off this experience as one-off or not scalable, but it is quite real and we predict recurrence in your clinical and scientific work in the years ahead. The real pioneers of this shift were the Odones (The Myelin Project and adrenoleukodystrophy work featured in "Lorenzo's Oil"), the Crowleys (Pompe disease, highlighted in "The Cure" and "Extraordinary Measures"), and the Marguses (Genome Bridge at Broad and leaders in ataxia-telangiectasia research), but they were just the beginning. More patients and parents are teaching themselves genetics and subtle nuances of medicine (e.g., glycobiology). This is not simple patient advocacy or grantsmanship. Those activities are present, but they are minor compared to the larger macro trend we are seeing in the democratization of rare disease discovery and treatment.

Although one of us comes from business and the other from academia, we share a common bond in information technology. We believe that information technology is transforming medical science. There are several information technology best practices in other industries that we can apply to the NGLY1 team:

- Share early, share often. Collaborate across institutions, disciplines, patients, and parents. We recognize that this is easier said than done, but it is essential. Share data. Share negative results. For findings too small to be publishable, turn to the Web and publish them in short blog posts. Get the information out there. When Stanford and Baylor did whole-genome sequencing in the Fall of 2011 on an NGLY1 patient, they produced eight candidate genes (including NGLY1) to explain the phenotype. Stanford and Baylor shared their list of variant candidates quickly. It was a dialogue with clinicians, other researchers, parents, and bioinformatics experts. The teams triaged the list and worked systematically to close certain doors. Stanford and Baylor initially thought another gene was the main culprit, but functional work did not support this. Both teams continued to refine until NGLY1 was confirmed via functional assays. The lesson is that genetics is an iterative process.

- Balance the bottom-up with the top-down. We recognize that much of science happens "bottom-up," with openended investigations uncovering basic truths. Balance this approach with "top-down," measurable goals that are grounded in patient's needs. We know science takes random turns and there will be setbacks and pitfalls, but no successful business has been built without measurable goals and targeted objectives. Strike a balance.

- Move fast and break things. This motto is common in Silicon Valley, but has been popularized by Facebook CEO Mark Zuckerberg. Genetics, like any branch of medicine, is justly conservative when it comes to patients ("Do no harm"). We do not suggest that you should be less thorough or loosen protocols. We simply suggest that you take risks to make discoveries, even if ideas or assays fail along the way. Failure is good. We can all build upon those failures if we share.

We leave you with this conclusion outside of NGLY1. In order to diagnose patients, we must admit the limitations of our medical knowledge. Sometimes the best ideas come from individuals "outside the box" (i.e., patients and parents). Pay special attention to the smallest details (e.g., lack of tear production); sometimes the least likely gene candidate is the answer. Last but not least, thank you. What you do is truly remarkable. When you have a bad day in the clinic or the laboratory, please remember that there are patients and parents out there who you do not know and who are dreaming of finding you, supporting you, and counting on you.

\section{DISCLOSURE}

The authors declare no conflict of interest.

\section{REFERENCES}

1. Enns GM, Shashi V, Bainbridge M, et al. Mutations in NGLY1 cause an inherited disorder of the endoplasmic reticulum-associated degradation pathway. Genet Med 2014; this issue (gim.2014.22).

2. Need AC, Shashi V, Hitomi Y, et al. Clinical application of exome sequencing in undiagnosed genetic conditions. J Med Genet 2012;49:353-361.

3. Might M. Hunting down my son's killer. http://matt.might.net/articles/my-sonskiller/ Accessed December 25th, 2013. 